\title{
Performance Evaluation of Interactive Video Streaming over WiMAX Network
}

\author{
Sanam Narejo ${ }^{1}$, Farzana Kulsoom ${ }^{2}$, M. Moazzam Jawaid ${ }^{3}$ \\ ${ }^{1}$ Departement of Electronics and Telecommunication, Politecnico Di Torino, Italy \\ ${ }^{2}$ Departement of Electrical, Computer \& Electronics, University of Pavia, Italy \\ ${ }^{3}$ Department of Electrical \& Electronics Engineering, City University, United Kingdom
}

\begin{tabular}{l} 
Article Info \\
\hline Article history: \\
Received Jun 9, 2016 \\
Revised Nov 20, 20 \\
Accepted Dec 11, 2016 \\
\hline Keyword: \\
Frames per second \\
Pixel resolution \\
QoS \\
Video streaming \\
Wireless network
\end{tabular}

Article Info

Article history:

Received Jun 9, 2016

Revised Nov 20, 2016

Keyword:

Frames per second

QoS

Wireless network

\begin{abstract}
Nowadays, the desire of internet access and the need of digital encodings have influenced quite a large number of users to access high quality video application. Offering multimedia services not only to the wired but to wireless mobile client is becoming more viable. In wireless medium, videostreaming still has high resource requirements, for example, bandwidth, traffic priority, smooth play-backs. Therefore, bandwidth demands of these applications are far exceeding the capacity of $3 \mathrm{G}$ and Wireless Local Area Networks (LANs). The current research demonstrates the introductory understanding of the Worldwide Interoperability for Microwave Access (WiMax) network, applications, the mechanisms, its potential features, and techniques used to provide QoS in WiMAX, and lastly the network is simulated to report the diverse requirements of streamed video conferencing traffic and its specifications. For this purpose two input parameters of video traffic are selected, i.e, refresh rate, which is monitored in terms of frames per second and pixel resolutions which basically counts the number of pixels in digital imaging. The network model is developed in OPNET. Different outcomes from simulation based models are analyzed and appropriate reasons are also discussed. Apart from this, the second aim of the current research is to address whether WiMAX access technology for streaming video applications could provide comparable network performance to Asymmetric Digital Subscriber Line (ADSL). For this purpose network metrices such as End to End delay and throughput is taken into consideration for optimization.
\end{abstract}

Copyright @ 2017 Institute of Advanced Engineering and Science. All rights reserved.

Corresponding Author:

Sanam Narejo,

Department of Electronics and Telecommunication,

Politecnico Di Torino, Italy.

Email: sanam.narejo@polito.it

\section{INTRODUCTION}

Streaming of real time applications over any wireless network is basically a key issue. It faces many challenges due to uncertainty in the wireless network and its dynamic environment, which actually causes link errors and high packet loss ratios [1].

Interesting technical challenges arise when un-predictable nature of the wireless channel meets the requirements of high data rate and low latency of video transport. Video streaming intuitively requires a steady flow of information and delivery of packets with some acknowledgement within a certain time frame. However, wireless networks have difficulty to provide such a service reliably and this results as a jerky, frozen and pixilated video [2]. As demands for broadband services are growing spectacularly, and as the required transmission bandwidth increases. Moreover, it is expected that future technologies such as TV-ondemand, digital library access, video conferencing and fast Internet access will be all IP-based. As far as the 
WiMAX is concerned, it is likely to be one of the hottest wireless broadband technologies [3]. Due to the growing requirements forced by the enhanced multimedia services on bandwidth availability and everpresent connectivity, access technology choice becomes a key part of each service provider's strategy.

With the rollout of the third generation cellular networks, 3G, the seek is already set towards the next generation. Future generation networks will be exemplified by variable and high data rates, quality of services and flawless mobility both within a network and between networks of different technologies and service providers. An important phase of the components in a global next generation network is standardization to allow vendor independence and interoperability.

A technology developed to accomplish these characteristics, standardized by IEEE, is 802.16 . It is commonly referred to as Worldwide Interoperability for Microwave Access (WiMAX). WiMAX is the endto-end network architecture built on the IEEE. 802.16 And alterations such as the IEEE 802.16e. WiMAX plan is to have high data rate, Quality of service (QoS), range, low cost to access wirelessly within a metropolitan area. The network structural design is developed by the WiMAX Forum, an interest group backed by technology companies, such as Intel, Fujitsu, Samsung, AT\&T, Alvarion, Wateen, and Motorola.

It uses Scalable Orthogonal Frequency-Division Multiple Access (SOFDMA) rather than Orthogonal Frequency-Division Multiplexing (OFDM). Additionally, it further employs two multiple duplexing schemes: Time Division Duplexing (TDD) and Frequency Division Duplexing (FDD) and provides bandwidth to hundreds of Internet subscribers with frequency band frame $10 \mathrm{GHz}$ to $66 \mathrm{GHz}$.

Video streaming enables viewers to start video playback while the content is being downloaded. The two dominant sources for video streaming traffic in the internet space are Netflix and YouTube [4]. In this paper, we study the network characteristics of the two most popular video streaming services, Netflix and YouTube. We also present that the streaming strategies vary with the type of the application (Web browser or native mobile application), and the type of container (Silverlight, Flash, or HTML5) used for video streaming [5].

Streaming-Video applications adopt more lenient QoS requirements because they are not delay sensitive as the video can take several seconds to queue up and are largely not jitter sensitive because of application buffering. Although, Streaming-Video might contain valuable content, such as e-learning applications or multicast company meetings, in which case it requires service guarantees. However, video conferencing is interactive-video streaming. When provisioning QoS for Interactive-Video, i.e, video conferencing traffic, the following guidelines discussed subsequently are recommended. Loss should be no more than $1 \%$. However, video streaming whether unicast or multicast can tolerate minimum loss of $5 \%$. Latency should be no more than 4 to 5 seconds depending on the video application's buffering capabilities for video streaming. One-way latency should be no more than $15 \mathrm{~ms}$. The limited jitter should be no more than $30 \mathrm{~ms}$. There are no significant jitter requirements.

Recently, various mobile broadband service providers have started a TV service over mobile WiMax networks, which is expected to be expanded in near future [6], [7]. Although, maintaining top quality of service is one of the main challenges for these applications. Video surveillance applications have experienced an increase in demand over the last decade. The researchers in [8] have attempted to adjust the multipurpose. The contributions of their research are simplified service architecture, packet-aware bandwidth request mechanism and packet-aware scheduling algorithms for dedicated video surveillance application with real-time uniform video traffic.

WiMAX, with its deployment ease and adaptive high bandwidth capacity, broaden the reach of IMS and represents the fastest growing access technology in the market today. One of the main advantage of it, is the large degree of flexibility which is supported by a wide range of traffic classes with different characteristics and QoS requirements [9], [10]. Along with such growth, to realize the tradeoffs of moving to WiMAX, it is desirable to compute application performance across this technology using bandwidth intensive application loads such as video streaming. Consequently, in this research, for evaluation and understandings of multimedia traffic specifically video contents, the WiMAX network is taken to be the most desirable one. The work mainly focuses on analysis and evaluation of multimedia traffic by simulating the video contents like IPTV and other rich-video applications over WiMAX network.

The rest of the manuscript is organized as follows. Section 2 provides the details on the adopted research methodology along with the simulated architecture modeling for the current project. Additionally, the corresponding details for parameter settings are also provided in this section. Section 3 deals with the comprehensive analysis of the obtained results with appropriate reasons for the achieved observations. Section 4 provides conclusion with a direction for possible future work.

\section{RESEARCH METHOD}

A rapid method for designing large WLAN, MANET, or custom wireless technology-based networks is to make use of the wireless network deployment wizard in Opnet modeler. By using this wizard 
one can configuring key aspects and features of networks rapidly, and easily. Networks can be customized to contain a great level of detail for example node layout, mobility, altitude, transmission power, and more. The developed model in this study contains three major parts. There is one server which is connected with two subnets, one is wired and one is wireless. The major steps followed in order to develop the network model for our study are presented in Figure 1.

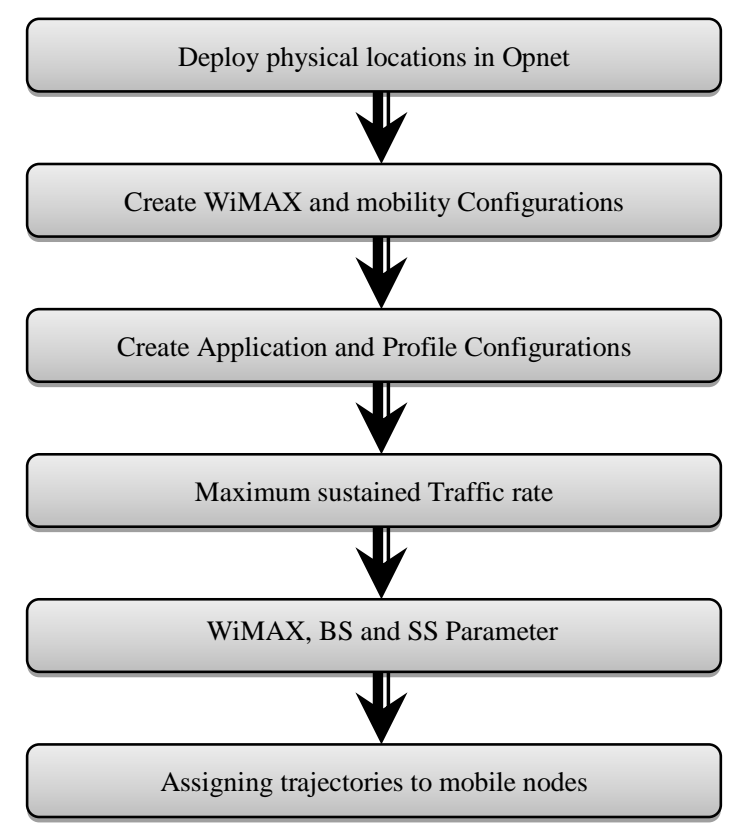

Figure 1. Flow of Simulation Model

\subsection{Developed Network Model}

For analysis purpose, we consider a scenario based on three subnets. Physically, the simulated model covers area of some random cities on the map. The proposed model is presented in Figure 2 . The project model consists of three subnets, covering area of some cities in Pakistan. The server is located at Rawalpindi city and two networks one wired and one wireless are located at the Hyderabad city in Sindh province of Pakistan. The model is shown in Figure 3. The Rawalpindi server is connected to both DSL network and wireless network at city Hyderabad. The topology implemented for wireless was WiMAX technology. The whole network was connected through OC3 fiber optic line, which served as backbone to the network model.

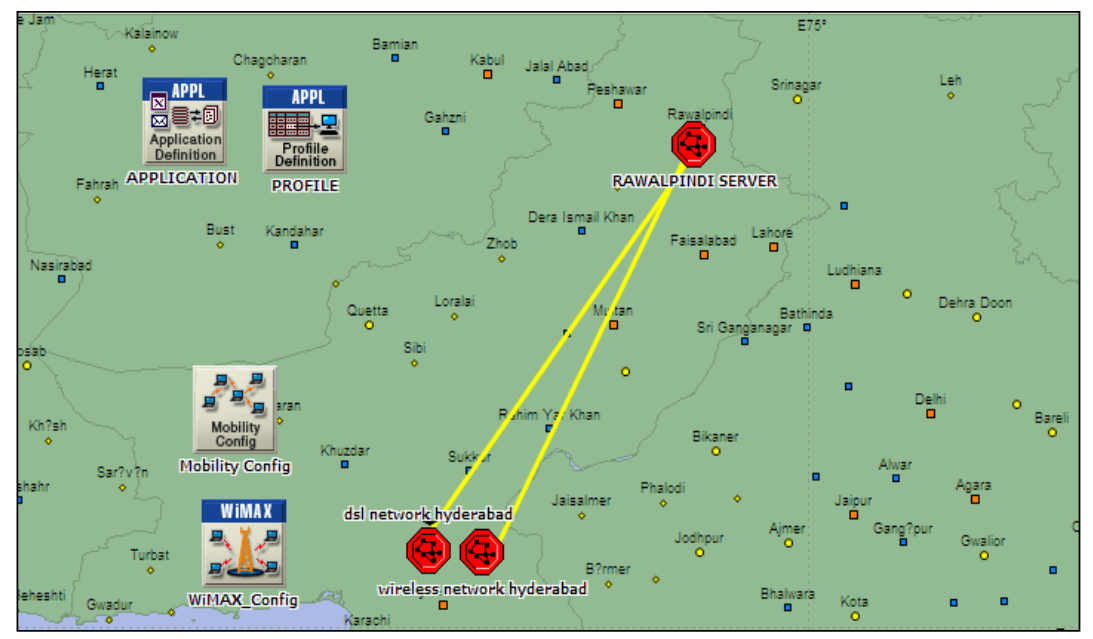

Figure 2. Simulation Model of Research Project 


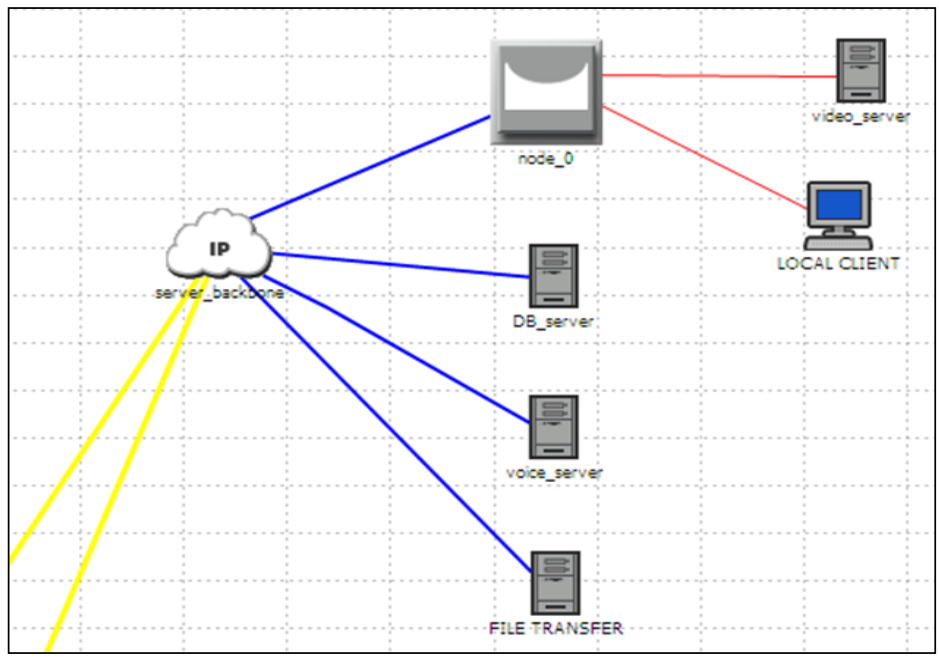

Figure 3. Simulation Model Rawalpindi Server Subnet

\subsection{Wireless Subnet}

This wireless subnet is of greater importance in the entire project model. As it is clearly visible from Figure 4, that this picture presents an expanded view of WiMAX network covering the whole areaof Hyderabad. The figure shows that there are seven cells in the WiMAX network. Each cell is equipped with a Base Station (BS) to control uplink and downlink connections. There are in total three Subscriber Station (SS) in each cell. Out of which the two are mobile nodes and one is fixed node.

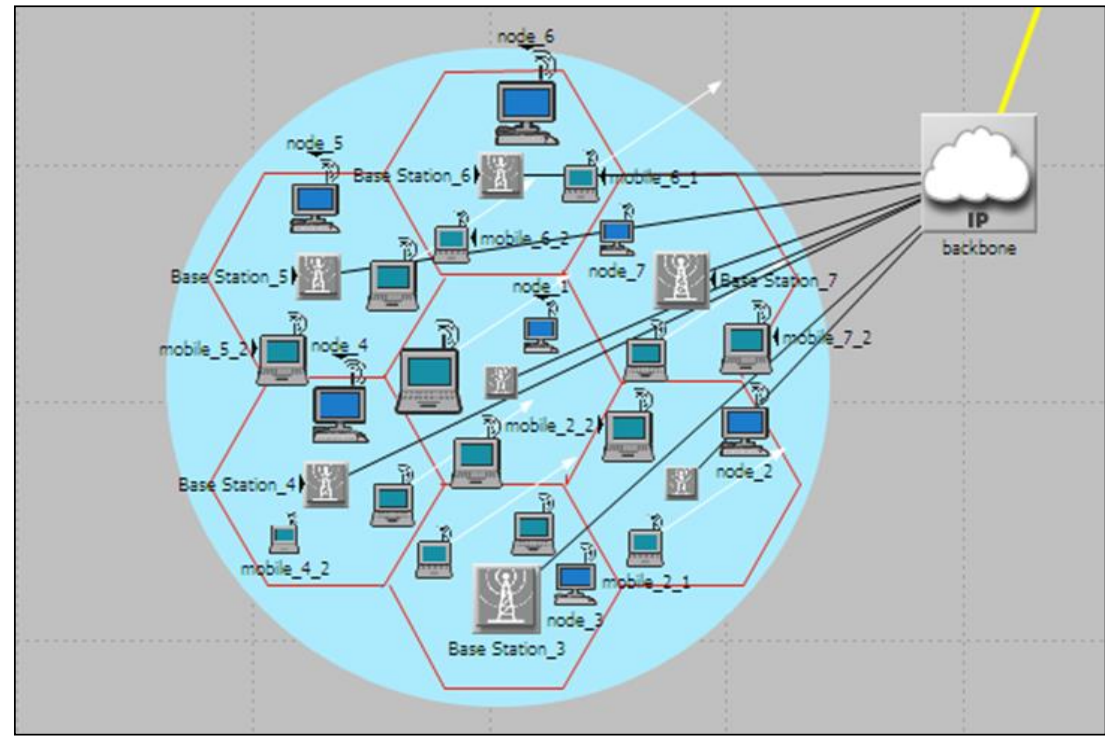

Figure 4. Simulation Model Wimax, Hyderabad Wirelss Subnet

In order to transmit the video traffic to and from the source and destination, few more steps were followed in chronological order. Once the wireless network topology is deployed in OPNET, the parameters of the networks are needed to be initialized. Therefore, we have to set some preferences in WIMAX configurations. In order to perform this, an appropriate Media Access Control (MAC) service class is selected as seen in Figure 5 and Figure 6. In order to assign the trajectories to mobile nodes mobility config attributes are set as shown in Figure 6. 
The next successive step is to create a traffic application of streamed video conferencing and deploy its appropriate profile over the network model. At the end BS and SS attributes are also set to appropriate values.

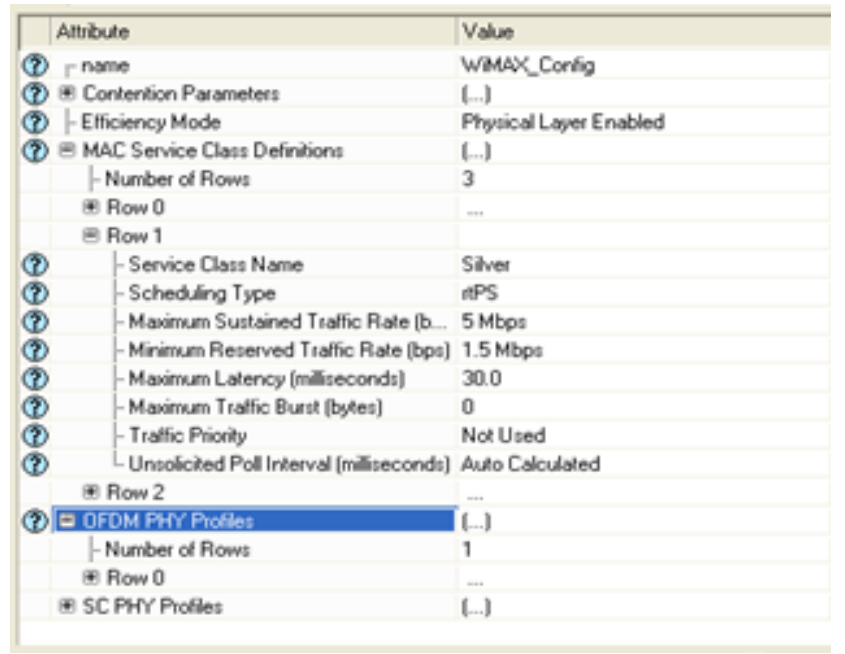

Figure 5. Attributes of Wimax Config from OPNET Modeler

\begin{tabular}{|c|c|c|}
\hline & Altribute & Value \\
\hline (?) & name & Mobility Config \\
\hline (?) & Mobility Modeling Status & Enabled \\
\hline (?) & $\Theta$ Random Mobility Profiles & (...) \\
\hline & Number of Rows & 4 \\
\hline & $\oplus$ Default Random W/aypoint & $\ldots$ \\
\hline & $\oplus$ Random Waypoint [Record Trajectory] & $\ldots$ \\
\hline & $\$$ Static & $\ldots$ \\
\hline & ๑ Random Waypoint (Auto Create)_1 & $\ldots$ \\
\hline
\end{tabular}

Figure 6. Attributes of Mobility Config from OPNET Modeler

\section{RESULTS AND ANALYSIS}

The observed results are on the basis of two input parameters of video traffic such as refresh rate and pixel resolution. By analyzing the obtained graph statistics, best specifications are concluded as a requirement of streamed video conferencing traffic. Both of the considered parameters are variated with their appropriate values. The number of times a display's image is repainted or refreshed per second is known as refresh rate. The refresh rate is expressed in terms of frames per second. One can easily alter the refresh rate in the display properties. However, if one tries to change the refresh rate to a setting that the display hardware or video card not supports, correspondingly, the image becomes distorted or the display goes blank. It is recommended to consult the display. The second chosen parameter is pixel resolution. The resolution is often used as a pixel count in digital imaging.

The pixel can be defined as an independent picture element and is usually articulated in terms of the product of width and height, which forms a picture frame. This explanation is one dimensional description used to define an image. However, a video stream is generally composed of multiple picture frames that are continuously displayed in a sequence of one after the other. The frame rate of streamed video is computed in terms of frames per seconds. Infact, this frame rate represents the motion aspect of the video stream.

In order to investigate the best video graphics modes for robust QoS in WiMAX network three different video resolutions were considered. Moreover, on each specified resolution three motion aspects as mentioned in Table 1 are examined. The mentioned Table summarizes the variable input parameters along with possible outcomes. As seen from the table that the most of the received video traffic in all cases is mostly around $50 \%$ or less. This indicates that packet loss is a noteworthy issue while conducting the video 
conference. It is certain that the obtained results indicate trade-offs between quality and delay. It is a known fact that various parameters affect the QoS of WiMAX such as Packet loss, end to end delay and throughput.

It is clearly visible from the table that when video conferencing traffic was sent with the higher resolution video settings, i.e, $352 * 240$, the network was not capable enough to transmit it. In response to this nothing was received at the destination. This observation was similar for the three considered motion aspects rates. The table further demonstrates that the substantial amount of traffic was received when the resolution settings were taken as $128 * 240$ at medium resolution. This interpretation is further confirmed with the help of WiMAX load, throughput and delay for the motion aspect $15 \mathrm{fps}$ at a specified medium resolution as shown in Figure 7(a) and (b) .

Table 1. Performance Analysisof Video Traffic Sent and Received

\begin{tabular}{ccccc}
\hline $\begin{array}{c}\text { Input Parameters } \\
\text { RESOLUTION } \\
\text { (No: of pixels) }\end{array}$ & $\begin{array}{c}\text { Refresh Rate } \\
\text { (fps) }\end{array}$ & $\begin{array}{c}\text { Video Traffic sent (No. } \\
\text { of Packets/Sec) }\end{array}$ & $\begin{array}{c}\text { Possible Outcomes } \\
\text { Video Traffic received } \\
\text { (No. of Packets/Sec) }\end{array}$ & $\begin{array}{c}\text { Traffic received } \\
(\%)\end{array}$ \\
\hline $\begin{array}{c}\text { HIGH RESOLUTION } \\
(352 * 240)\end{array}$ & 10 & 20 & - & $0 \%$ \\
& 15 & 60 & - & $0 \%$ \\
LOW RESOLUTION & 30 & 150 & - & $0 \%$ \\
$(128 * 120)$ & 10 & 60 & 20 & $50 \%$ \\
& 15 & 120 & 30 & $50 \%$ \\
MEDIUM RESOLUTION & 30 & 20 & 10 & $8.3 \%$ \\
$128 * 240$ & 10 & 30 & 10 & $50 \%$ \\
& 30 & 180 & 15 & $50 \%$ \\
\hline
\end{tabular}
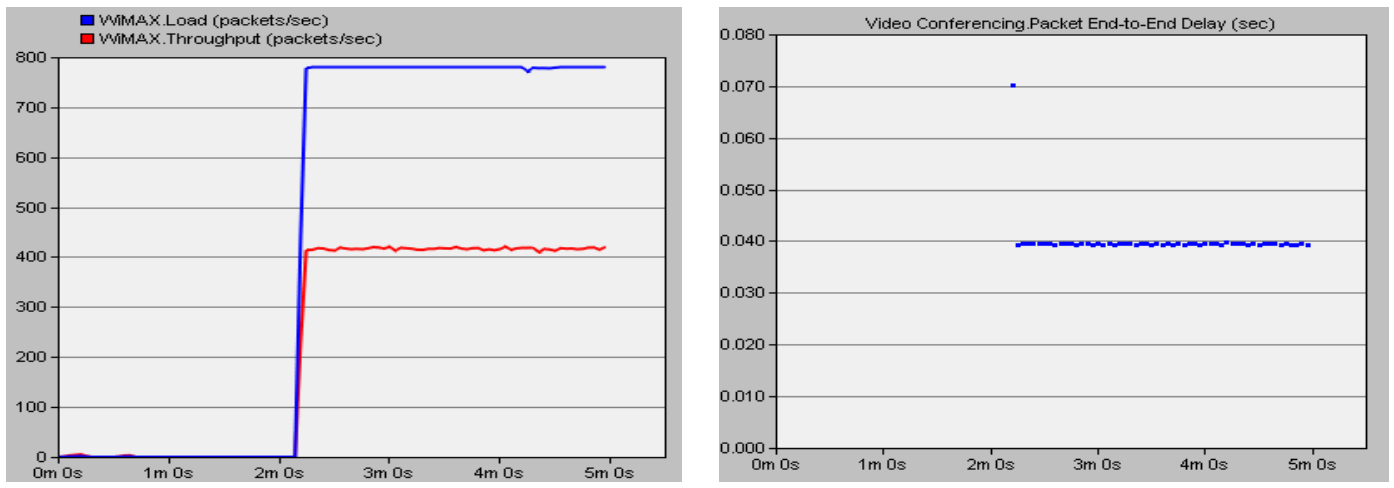

Figure 7. (a) Load and throughput for $15 \mathrm{fps}$ at medium resolution (b) Packet end-to-end delay for $15 \mathrm{fps}$ at medium resolution

The Digital Subscriber Line (DSL) network is developed so that video conferencing streamed traffic can be analyzed on this wired technology in response to compare the performance of both wire and wireless technologies and to further verify whether WiMAX competes to its alternate wired broadband i.e. ADSL or not. In order to develope a simulated DSL network model seven different types of work stations for instance, campus, remote office, home, building, etc. These substations are present over different locations, which can be seen in the figure. As shown in figure 8 that work stations from all seven locations are connected to access router through DSLAM.

In order to perform the comparative analysis with strong evidence, we refer to the graphs presented in the above figures of DSL and WiMax node statistics. While focusing on the DSL node statistics, it is 
obvious from the Figure 9(a) and (b) that in DSL network, when traffic starts receiving at node, initially the traffic is received at Constant Bit Rate (CBR) with very less variations and fluctuations in the receiving rate. But, as the simulation time further increases, the network traffic over head increases. This results in congestion. Due to this congestion, packets starts dropping as it can be seen in Figure 9(b). As far as WiMAX network is concerned, the receiving is at the rate of 15 packets/sec. A lot of variations can be seen in Figure 10(b), which demonstrates the rate of the received traffic. This variation is due to its dynamic nature of wireless medium. In response to provide comparison between both technologies, the obtained delay of both networks is further examined. As seen in figure 11(a) and (b), DSL node encompasses more delays up to 15 seconds, whereas the delay in WiMAX node is significantly less, even less as 0.022 seconds. Additionally, it is also perceived from the results that the delay at DSL node might increases linearly as the simulation time is increased.

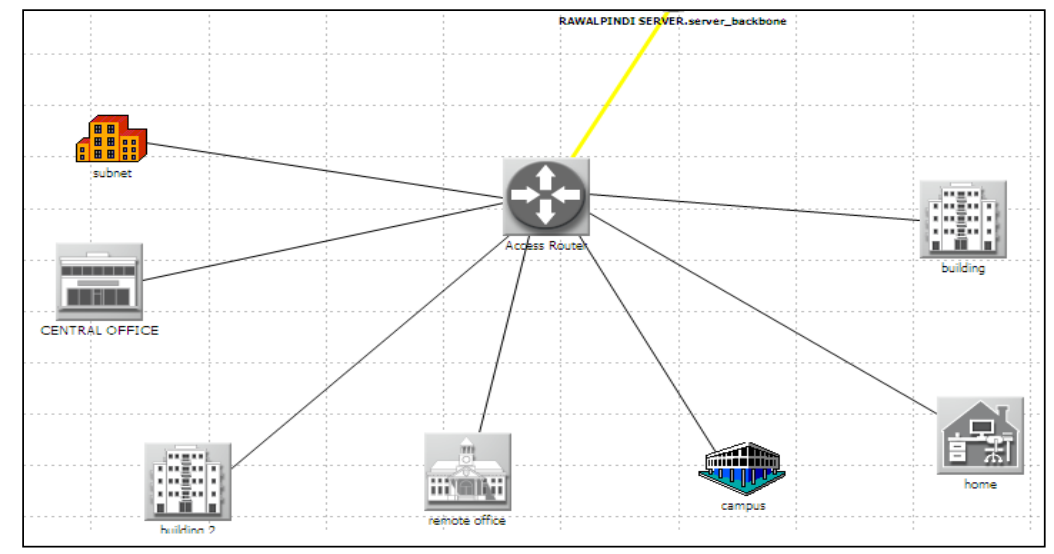

Figure 8. Simulation Model DSL Network
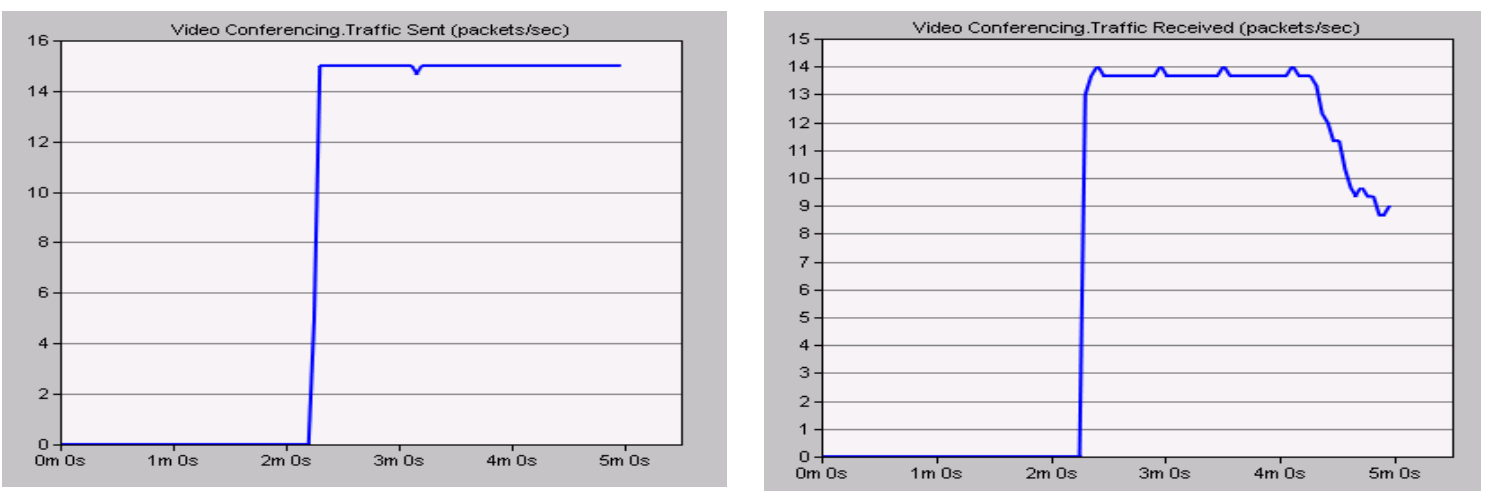

Figure 9. DSL node statistics showing no: of packets (a) sent and (b) received
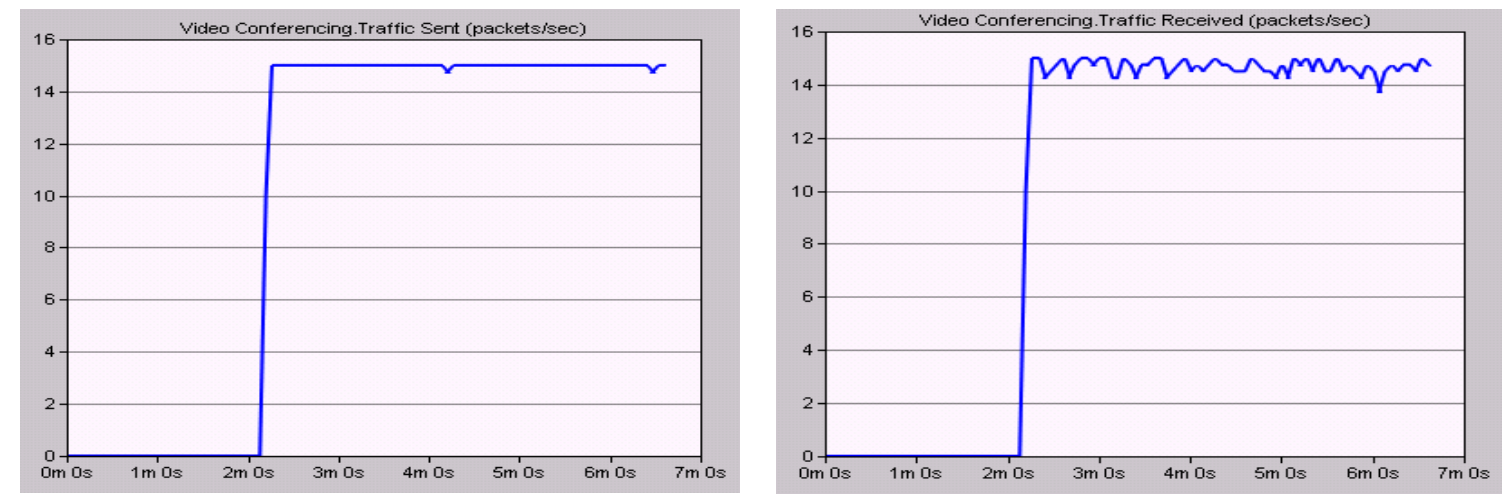

Performance Evaluation of Interactive Video Streaming over WiMAX Network (Sanam Narejo) 
Figure 10. WiMAX node statistics showing no: of packets (a) sent and (b) received
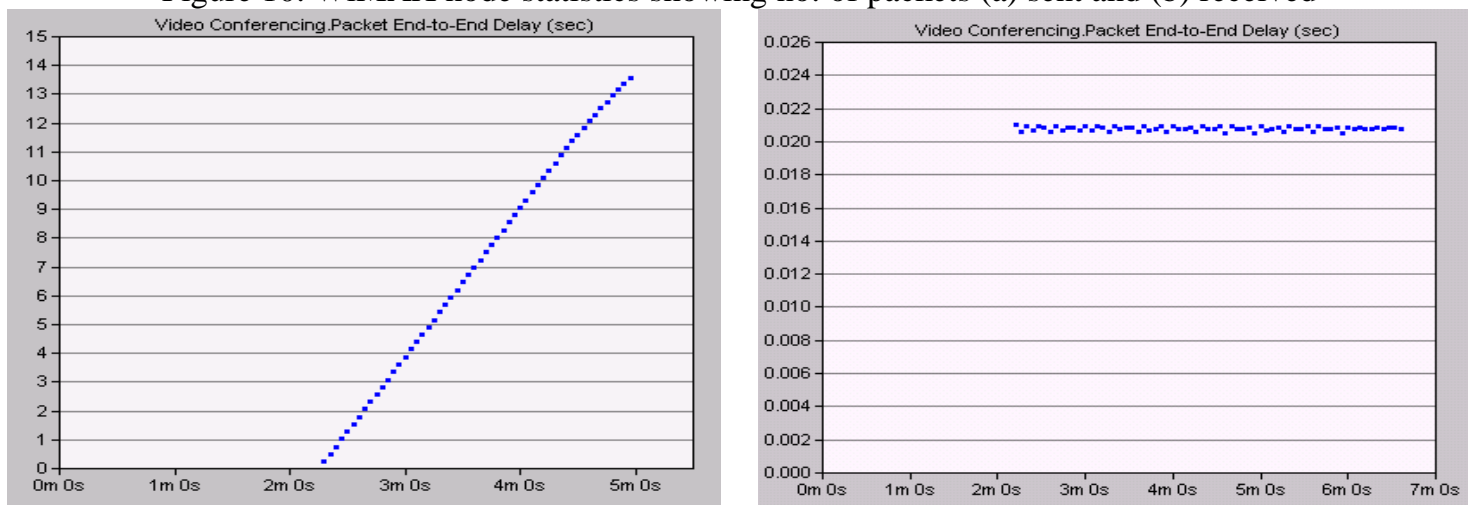

Figure 11. Packet End to End Delay (a) DSL and (b) WiMAX

\section{CONCLUSION}

In this research work, for addressing the diverse requirements of streamed video traffic over WiMAX networks, two parameters of video quality refresh rate and pixel resolutions were chosen. Subsequently, the second aim of this research was to address whether WiMAX access technology for streaming video applications could provide comparable network performance to ADSL. Specific conditions and parameter settings were considered in this study. However, there may be variations in the results if these input conditions and parameters are altered.

According to the observations found in the current study, for video traffic communication generally, $30 \mathrm{fps}$ or approximately more than $20 \mathrm{MHz}$ bandwidth is needed. However, on the basis of observed findings and simulation results $30 \mathrm{fps}$ rate was not applicable in the WiMAX network for video conferencing traffic. Although, the obtained results demonstrate the best statistics of video conferencing traffic at the rate of $15 \mathrm{fps}$ with a pixel resolution taken as $128 * 120$ in the simulated scenario as mentioned in the methodology. It was also identified that if the rate is considered below $10 \mathrm{fps}$ this results as transmission of a jerky video. Moreover, if the rate is reduced further, for instance below $5 \mathrm{fps}$, the transmitted video is in the form of playing a slide show.

In response to the second objective of our study, WIMAX proved to be a strong contender that competes its alternate DSL technology with its promising behavior as seen in the Results section. Apart from this, the delay in WiMAX network traffic was appreciably much lower than DSL network traffic. WiMAX standard delivers prompt-speed broadband internet access above a wireless link over relatively long distances. Number of applications can employ this standard, comprising "last mile" broadband links, hotspots and cellular backhaul, and prompt-speed enterprise linkages for business. WiMAX supports P2P and PMP operation modes and is an alternative to cable and DSL providing broadband access to sites wherever wired connection would be hard or costly.

For further possible extension of present research, different video quality perceptions and specifications should be taken into consideration and further to be analyzed on real time video streaming traffic patterns.

\section{REFERENCES}

[1] B. Kaarthick, V. J.Yeshwenth, N.Nagarajan, "Rajeev Performance analysis of Video Conferencing and Multimedia application Services over WiMAX”, 2009 IEEE International Advance Computing Conference (IACC 2009)

[2] Emir Halepovic, Majid Ghaderi, Carey Williamson :Application Performance on a WiMAX Network Sep-2008

[3] Awan, Khalid Mahmood, Abdul Hanan Abdullah, and Khalid Hussain. "Additional Resource Allocation for improving Fairness in WiMAX", TELKOMNIKA (Telecommunication Computing Electronics and Control) 12.2 (2014): 455-464.

[4] Global internet phenomena report. Technical report, Sandvine, 2011

[5] Rao A, Legout A, Lim YS, Towsley D, Barakat C, Dabbous W, "Network Characteristics of Video Streaming Traffic", In Proceedings of the Seventh Conference on emerging Networking EXperiments and Technologies 2011 Dec 6 (p. 25). ACM.

[6] Sharangi, Somsubhra, Ramesh Krishnamurti, and Mohamed Hefeeda, "Streaming scalable video over WiMAX networks", Quality of Service (IWQoS), 2010 18th International Workshop on IEEE, 2010. 
[7] Sharangi, Somsubhra, Ramesh Krishnamurti, and Mohamed Hefeeda, "Energy-Efficient Multicasting of Scalable Video Streams over Wimax Networks", IEEE Transactions on Multimedia 13.1 (2011): 102-115.

[8] Suherman, S., \& Al-Akaidi, M. (2013), “Adjusting WiMAX for a Dedicated Surveillance Network”, International Journal of Electrical and Computer Engineering, 3(4), 492.

[9] Narejo, Sanam, et al. "A parametric QoS analysis of WiMAX Scheduling Services." Sci. Int. (Lahore) (2013): 10131018.

[10] Lygizou A, Xergias S, Passas N, "Video Traffic Prediction for Improved Scheduling in Joint Wimax/Satellite Networks", In2012 8th International Wireless Communications and Mobile Computing Conference (IWCMC) 2012 Aug 27 (pp. 1017-1022). IEEE. 